\title{
Pharmacologic Pain Treatment for Adiposis Dolorosa: A Case Report
}

\author{
Ankur A. Patel, BS ${ }^{1}$, Arpit A. Patel, DO ${ }^{2-4}$, and Devang M. Padalia, MD
}

Dercum's disease, also known as adiposis dolorosa, is a rare pain syndrome caused by one or more painful lipomas. We present an educational case of Dercum's disease resulting in debilitating pain. An 80-year-old male developed multiple painful lipomas within a span of 24 months. The patient underwent surgical excision of the larger lipomas on the anterior thorax and thoracolumbar region, but continued to complain of diffuse pain. The pain at the post-resection sites was consistent with nociceptive-related pain from acute surgical intervention, but he also continued to complain of burning, neuropathiclike pain in the surrounding areas of the lipomas. The patient received minimal benefits from nonsteroidal anti-inflammatory drugs (NSAIDs) and adjuvant therapy, including neuromodulating agents. The patient was started on progressively larger doses of oral opioids which resulted in no relief. The medical regimen was changed to transdermal fentanyl and monitored closely by the pain management physician. Fentanyl was used to capitalize on the highly lipophilic nature of this medication, which was hypothesized to address the fatty tumor-related pain. The patient reported pain reduction of more than $50 \%$ and thus was able to continue activities of daily living with an improved quality of life.

Key words: Adiposis dolorosa, Dercum's disease, lipoma, lipophilic, opioid, pain management
Dercum's disease (adiposis dolorosa), first identified by neuropathologist Francis Xavier Dercum in 1888 , is a rare disorder consisting of multiple painful lipomas. These lipomas most commonly occur in the upper arms, upper legs, and trunk region of obese, female individuals. The exact etiology is unknown, but many have hypothesized that it is most likely due to an autoimmune disorder against healthy fat tissues. The clinical presentation typically varies, and as a result, the condition is often misdiagnosed as fibromyalgia or a related widespread pain syndrome. It is imperative that clinicians are familiar with Dercum's

From : ${ }^{1}$ Edward Via College of Osteopathic Medicine; ${ }^{2}$ Florida Pain Medicine; ${ }^{3}$ Department of Interventional Pain Medicine, University of South Florida; ${ }^{4}$ Department of Physical Medicine \& Rehabilitation, University of Central Florida; ${ }^{5}$ Department of Interventional Pain, H. Lee Moffitt Cancer Center \& Research Institute

Author for correspondence: Ankur A. Patel, BS

Address: Edward Via College of Osteopathic Medicine, 2265 Kraft Dr, Blacksburg, VA 24060

E-mail: ankurp416@gmail.com disease in order to promptly diagnose and manage the patient's pain accordingly. There are many treatment modalities available to help manage the symptoms, although a comprehensive approach is recommended to provide the most adequate relief.

\section{CASE PRESENTATION}

An 80-year-old non-obese male with a past medical history of Crohn's disease presented to the interventional pain service with a chief complaint of diffuse pain in the anterior thorax and upper and lower back. After multiple workups at leading research institutions around the US, he was ultimately diagnosed with Dercum's disease. This patient underwent surgical excisions of multiple painful, fatty tumors on the anterior thorax, abdomen, and thoracolumbar region. Post-operatively, the patient continued to have pain at the surgical resection sites, as well as in areas surrounding the unresected lipomas. The patient rated pain severity within the anterior thorax, abdomen, and thoracolumbar regions as the highest rating of $10 / 10$. He described it as a burning, deep achy sensation and noted minimal relief from NSAIDs and 
adjuvant therapy. The patient's primary care physician then prescribed progressively increasing doses of oxycodone/acetaminophen $10 \mathrm{mg} / 325 \mathrm{mg} 4$ times a day, which were ineffective in managing the pain. The patient was then referred to a pain management specialist for further evaluation and treatment options. Alternative treatment methods for Dercum's disease including methadone, intravenous lidocaine, and ketamine were not considered safe medications in this patient secondary to his history of cardiac disease and conduction abnormalities. At a low dose, methadone was trialed with no relief. Patient also failed extended release morphine doses. He was concomitantly started on multiple neuromodulating agents including duloxetine $60 \mathrm{mg}$ daily and gabapentin $800 \mathrm{mg} 4$ times a day to help provide more comprehensive pain relief. Unfortunately, the patient continued to have significant pain. Upon literature review and collaboration from the providers at the pain medicine practice, the patient's opioid was switched to fentanyl transdermal 72 hours $50 \mathrm{mcg} / \mathrm{hr}$. Only after starting transdermal fentanyl did the patient report a significant pain reduction of greater than $50 \%$ with improved quality of life.

\section{DISCUSSION}

Dercum's disease is a rare disorder of painful fatty tumors associated with obesity. Historically, this condition has been more prevalent in postmenopausal women; however, a recent survey of 110 participants indicated that $85.7 \%$ of female patients who developed Dercum's disease were premenopausal (1). Although women are most commonly affected, 16\% of reported cases are men (2). Additionally, Dercum's disease can occur in patients who are not obese, as was the case with this patient. The upper arms, elbows, stomach wall, buttocks, thighs, and knees are regions that are most commonly affected (3).

Roux and Vitaut proposed 4 cardinal symptoms of Dercum's disease in 1901: multiple painful, fatty masses, generalized obesity, weakness and susceptibility to fatigue, and psychiatric manifestations (4). However, all 4 of these cardinal symptoms are rarely found together in a patient with Dercum's disease. Obesity and painful adipose masses are the 2 symptoms that manifest into this condition most often.
The pain associated with this condition is thought to be due to the compression of the peripheral nerves by the subcutaneous adipose tissues. As the tissue enlarges, it disrupts the nociceptors and afferent axons, which results in pain (3). Other theories include decreased subcutaneous blood flow to the adipose tissue due to a dysregulation in the sympathetic nervous system (3).

Due to the wide spectrum of presentation, Dercum's disease is classified into 4 categories:

- Generalized diffuse (painful adipose tissue without clear lipomas)

- Generalized nodular (painful adipose tissue and intense pain in and around multiple lipomas)

- Localized nodular (pain localized to, in, and around multiple lipomas)

- Juxtaarticular (painful solitary deposits of fat near large joints) (4).

Based on our patient's history, we believe he had a localized nodular form.

The pathogenesis of Dercum's disease is still unknown. There are many proposed hypotheses, of which the most prominent suggests a genetic link. A previously published case report found 5 family members with Dercum's disease with autosomal dominant inheritance (5). However, many other hypotheses are being considered, such as autoimmune or metabolic. More research is required to determine the definitive etiology of this condition.

Currently, there is no cure for the condition and it is treated symptomatically with surgery and/or pain management. Surgical excision has proven to significantly reduce pain for the patient, however, relapse in pain and recurrence of the disease often develops (3). Pain was evaluated in 53 patients with Dercum's disease over a 5-year period after liposuction. Subjective pain measurements significantly decreased after surgery, yet this pain relief was temporary as it decreased over the 5-year period (6). This study was inconclusive in determining whether the pain relief was a result of the surgery or other contributing factors. As observed, not all patients achieved pain relief from liposuction. For this reason, more research must be conducted to determine if liposuction has long-term efficacy (6). 
Due to the chronic nature of pain associated with Dercum's disease, many patients have an inadequate response to analgesic medications. Intravenous lidocaine has served as an effective option for patients with Dercum's disease. A case study described 2 patients feeling optimal pain relief after repeated treatments of intravenous lidocaine. Pain subsided for 8 and 25 days, respectively, for these patients (7). These patients were then treated with mexiletine, a Class IB antiarrhythmic that non-selectively blocks voltage-gated sodium channels. Even though this drug is mainly used to treat arrhythmias, it has shown to help treat refractory neuropathic pain. However, it must be used in caution due to the potential side effects of dizziness and confusion. These 2 patients had complete pain relief after the administration of mexiletine (7).

Another potential treatment option for Dercum's disease patients is ketamine, a noncompetitive antagonist of the N-methyl-D-aspartate (NMDA) receptor that has shown to provide relief for treatment-resistant neuropathic pain. In addition to this inhibition, it has anti-inflammatory effects at central sites (8). Ketamine has the potential to provide short- or long- acting analgesia; however, the side effect profile greatly limits the use of this drug. Side effects include hallucinations, cardiovascular stimulation, and confusion. Typically, patients tolerate the use of ketamine when it is given in conjunction with benzodiazepines, which controls the psychogenic side effects (8).

Opioids are the most widely used analgesics for pain syndromes. Delta $(\delta)$, kappa $(\kappa)$, and $\mathrm{mu}(\mu)$ are the 3 major opioid receptors present in the central nervous system as well as the peripheral nervous system (9). Peripheral opioid receptors are present on the peripheral sensory nerve terminals and they function similar to the opioid receptors found in the brain (9). These receptors are G-protein coupled and lead to in an inhibition of adenylyl cyclase, thus resulting in the overall decrease of the cyclic adenosine monophosphate (cAMP) concentration (10). This causes an increased conductance of potassium and decreased conductance of calcium at the synaptic junction. The changes in the conductance cause a reduction in the neuronal cell excitability, inhibiting the release of excitatory substances such as substance $P$ (9). Opioids are often categorized based on different properties including solubility and potency. Solubility is classified as lipophilic, hydrophilic, or intermediate. The lipophilicity of the opioid is determined by the oil-water partition coefficient, which is the difference between the partition coefficient in the oil phase and aqueous phase (11). The solubility of a chosen opioid should complement the targeted tissue in order to provide optimal analgesia. For this patient, targeting the opioid receptors and utilizing a potent lipophilic substance to specifically target the adipose tissue proved to be the most beneficial combination.

The high lipophilicity of fentanyl makes it the opioid of choice since in the transdermal form it can rapidly diffuse through the lipophilic epidermal tissue, but slowly diffuse into the hydrophilic dermal tissue and blood vessels (12). This results in continuous and greater pain relief than if immediate-release, less lipophilic opioids were to be used. The short duration of action of fentanyl is explained by rapid redistribution from the brain to skeletal muscle and fat. For patients who transitioned from oral opioids to fentanyl transdermal, it is important to note that therapeutic blood levels are not reached until 13-24 hours after the application of the patch, therefore another medication would be necessary for immediate pain control (13). The pharmacokinetics of fentanyl transdermal is an important consideration based on a patient's body habitus and age. For example, initially the transdermal patch absorbs in the upper skin layers. The therapeutic window is wide based on the medication's ability to store in fat and muscle. Elderly have poor fat stores, muscle wasting and alterations in hepatic clearance which could deem the medication ineffective at a similar dose to a younger patient. Since there are limited case reports using transdermal fentanyl as a treatment method for patients with Dercum's disease, additional research would further support the use of this medication in this patient population.

\section{CONCLUSION}

This case is an atypical clinical presentation of Dercum's disease. Most often, the lipomas are treated surgically, however, the chronic pain that forms is often debilitating and can be difficult to manage. There are a variety of opioids that can be used to manage such chronic pain syndromes, each of which has a different property and effect. Whether the opioid is hydrophilic or lipophilic can determine the efficacy on 
the affected target tissue. Therefore, careful consideration must be taken when deciding which opioid to use in order to attain optimal pain relief.

When managing pain from Dercum's disease, fentanyl is the opioid that should be considered early on because of its lipophilic properties and ability to concentrate in target adipose tissue. This results in a more effective of way of providing long-acting pain relief. Many case reports illustrate patients finding success with intravenous lidocaine and ketamine infusion. Providers who have not achieved adequate pain relief for their patients with these treatment options should consider transdermal fentanyl.

\section{REFERENCES}

1. Herbst $\mathrm{KL}$, Asare-Bediako $\mathrm{S}$. Adiposis dolorosa is more than painful fat. Endocrinologist 2007; 17:326-334.

2. Learning About Dercum Disease. National Human Genome Research Institute. Updated June 27, 2012. Accessed: January 27 , 2017 www.genome.gov/17516629.

3. Steiner J, Schiltz K, Heidenreich F, Weissenborn K. Lipomatosis dolorosa--a frequently overlooked disease picture. Nervenarzt 2002; 73:183-187.

4. Hansson E, Svensson H, Brorson H. Review of Dercum's disease and proposal of diagnostic criteria, diagnostic methods, classification and management. Orphanet J Rare Dis 2012; $7: 23$.

5. Cantu JM, Ruiz-Barquin E, Jimenez M, Castillo L, MacotelaRuiz E. Autosomal dominant inheritance in adiposis dolorosa (Dercum's disease). Humangenetik 1973; 18:89-91.

6. Hansson E, Svensson H, Brorson H. Liposuction may reduce pain in Dercum's disease (adiposis dolorosa). Pain Med 2011; 12:942-952.

7. Petersen P, Kastrup J. Dercum's disease (adiposis dolorosa) Treatment of the severe pain with intravenous lidocaine. Pain $1987 ; 28: 77-80$.
8. Niesters M, Martini C, Dahan A. Ketamine for chronic pain: Risks and benefits. Br J Clin Pharmacol 2014; 77:357-367.

9. Vadivelu N, Mitra S, Hines RL. Peripheral opioid receptor agonists for analgesia: A comprehensive review. J Opioid Manag 2011; 7:55-68.

10. Al-Hasani R, Bruchas MR. Molecular mechanisms of opioid receptor-dependent signaling and behavior. Anesthesiology 2011; 115:1363-1381.

11. Freye E, Levy JV. Opioids in Medicine: A Comprehensive Review on the Mode of Action and the Use of Analgesics in Different Clinical Pain States. Springer, Dordrecht, the Netherlands, 2008.

12. Nelson L, Schwaner R. Transdermal fentanyl: Pharmacology and toxicology. J Med Toxicol 2009; 5:230-241.

13. Facts about the fentanyl transdermal patch. Stanford School of Medicine. Accesed: February 03, 2017 https://palliative.stanford.edu/opioid-conversion/appendices/facts-about-the-fentanyl transdermal-patch. 